\title{
Diffuse $x$-ray scattering from thin films with defects
}

\author{
D. Bahr* and W. Press \\ Institut für Experimentalphysik, Universität Kiel, D-24098 Kiel, Germany \\ R. Jebasinski and S. Mantl \\ Institut für Schicht- und Ionentechnik Forschungszentrum Jülich GmbH, D-52425 Jülich, Germany
}

(Received 7 December 1994)

\begin{abstract}
Recent calculations predict a dramatic influence of interfaces on the scattering of $\mathrm{x}$ rays by single crystals with defects. The power law describing the diffuse scattering and the peak shape of the Bragg reflections depends on the boundary conditions of the crystal lattice. These theoretical results have been verified by grazing incidence diffraction of synchrotron radiation performed on thin layers of silicon and cobalt disilicide. By variation of the angle of incidence a depth profiling of the defect density has been performed.
\end{abstract}

\section{INTRODUCTION}

Defects in crystals create distortions of the lattice. The long range part of such a distortion field leads to diffuse scattering, e.g., of $\mathrm{x}$ rays, centered around the Bragg position. Its intensity distribution follows a power law $\propto q^{-2}$, where $\mathbf{q}$ is the wave vector measuring the distance from the Bragg reflection. If the defect concentration is not too high the shape of the Bragg reflection itself is only slightly modified. Assuming homogeneously distributed defects, the theory of bulk diffuse scattering is relatively straightforward. The influence of surfaces and interfaces can be neglected as long as the scattering is dominated by defects homogeneously distributed in the bulk. If a crystal has (i) a constrained geometry, if (ii) the defect distribution is varying on a sub- $\mu$ scale in the neighborhood of interfaces, or if (iii) the penetration of the $x$ rays is limited to the same length scale (grazing incidence diffraction), then the related relaxation of the displacement fields at the boundaries must be included. As predictions by Barabash and Krivoglaz ${ }^{1}$ show, the exact form of the boundary conditions decides whether the bulk exponent of the power law has to be modified and whether the Bragg reflection itself retains its shape. It is important whether the crystal lattice constant has to match that of the adjacent lattice or not, that is if shear forces and/or pressures are present or not. As the literature shows, both diffuse $\mathrm{x}$-ray scattering and grazing incidence diffraction are powerful tools of solid state physics. $^{2-4}$ Therefore these near surface effect of strains should be considered whenever diffuse scattering and grazing incidence diffraction are combined.

\section{GENERAL DESCRIPTION OF THE THEORY}

Barabash and Krivoglaz have analyzed three typical cases: (1) a depth distribution of defects in a semi-infinite crystal with relaxation at the surface, (2) defects in thin films with relaxation in the substrate and in the film, (3) defects in thin films with rigid coupling of the film to a substrate without defects (also known as pseudomorphic growth).

In the case of a bulk crystal the classical Huang scattering evolves: ${ }^{-12}$

$$
\begin{aligned}
I_{\text {Huang }}(\mathbf{Q}) \approx & c\left(\frac{\left|F_{h k l}\right|}{V_{c}}\right)^{2}\left(\frac{G_{h k l}}{q}\right)^{2} \\
& \times\left|\sum_{i=1}^{3} \frac{\mathbf{G}_{h k l}}{\left|\mathbf{G}_{h k l}\right|}{ }_{i} \tilde{g}_{i j}(\mathbf{q}) \frac{\mathbf{q}}{q_{l}} P_{j l}\right|^{2}
\end{aligned}
$$

$\tilde{g}_{i j}(\mathbf{q})$ is a tensor describing the elastic properties of a cubic crystal. ${ }^{7,8} c$ is the concentration of the defects and the defect symmetry is given by their double force tensor $P_{i j} . \mathbf{G}_{h k l}$ is the reciprocal lattice vector of the reflection $h k l, F_{h k l}$ the corresponding structure factor of the crystal, and $V_{c}$ the volume of the elementary cell. The Bragg reflection has $\delta$-peak shape in this kinematical approach. For experimental data this means the width of the reflections should be governed by the instrumental resolution.

If the sample solely contains near surface defects (case 1) the width of the Bragg reflection remains limited by the experimental resolution only. The diffuse scattering continues to follow the power law $q^{-2}$, but the distribution of the scattering around the reflection is quite different from that in the bulk case. As Grotehans et al. ${ }^{13}$ have shown, the power law changes to $q^{-1}$ if the penetration depth of the $\mathrm{x}$ rays is restricted to the near surface range.

In case 2 of Barabash and Krivoglaz both the peak shape and the power law behavior of the diffuse scattering need to be modified for the in-plane directions. In the surface plane the reflections are no longer resolution limited and broaden with a characteristic dependence on the defect concentration. The following expression results: 


$$
\begin{aligned}
& I(\mathbf{Q})=\frac{16 \pi^{2} N}{V_{c}}\left|F_{h k l}\right|^{2} \frac{\sin ^{2}\left(\frac{1}{2} q_{z} d\right)}{d q_{z}^{2}} \frac{1}{q_{i}^{2}} e^{-\frac{\pi\left(q_{x}^{2}+q_{y}^{2}\right)}{q_{i}^{2}}} \\
& q_{i} \approx 4 \pi l a \sqrt{c a_{0} / d^{3}}, \quad a=\frac{1+\sigma}{1-\sigma} L_{x x}
\end{aligned}
$$

(film thickness $d$, lattice constant $a_{0}$, third Miller index $l, q_{i}$ is the width of reflection which is about the same as the full width at half maximum: $\mathrm{FWHM}=\sqrt{\pi / \ln 2} q_{i}$, defect concentration $c$, structure factor $F_{h k l}$, tensor of internal strains $\mathbf{L}, \sigma=L_{x x} / L_{z z}$ Poisson ratio). The power law for the diffuse scattering, which is $q^{-2}$ in the bulk case, now is modified to $q^{-4}$. For $q_{r}>q_{i}\left(q_{r}=\right.$ $\left\{q_{x}, q_{y}\right\}$ : in the surface plane, $q_{z}$ : parallel to the surface normal) the authors get

$$
I_{\text {Huang }}=12(1-\sigma)^{2} N_{d} e^{-2 M_{L}}\left|F_{h k l}\right|^{2} \frac{Q_{z}^{2}}{d^{4} q_{r}^{4} q_{z}^{2}}\left|e^{i q_{z} d}-1\right|^{2} a^{2}
$$

[number of defects $N_{d}$, static Debye-Waller factor $\left.\exp \left(-2 M_{L}\right)\right]$. The authors have restricted themselves to elastic isotropic crystals and defects with cubic symmetry to render the problem analytically soluble. So the elastic properties of the crystal and the defect forces can be expressed by the Poisson ratio $\sigma$ and an isotropic strain $L_{x x}$. It should be emphasized that Eq. (3) depends in the same way on $q_{z}$ as Eq. (1). So the main differences appear for in-plane reflections. Extensions to noncubic defects have been elaborated by Belov and Kaganer. ${ }^{14}$

In case 3 there is no enhanced diffuse scattering around the Bragg reflections as for bulk crystals. The unstrained substrate strongly suppresses distortions by the defects in the film.

\section{EXPERIMENT}

Until now several experiments have dealt with the effect of near surface defects on Bragg reflections and diffuse scattering where the corresponding lattice planes are not perpendicular to the surface (e.g., Refs. 15-17). As Eqs. (2) and (3) show the influence is greatest for in-plane reflections, i.e., for grazing incidence geometry. Reference 13 has dealt with the near surface defects of as-implanted $\mathrm{Si}$ in such a geometry. In the nomenclature of Barabash and Krivoglaz this belongs to case 1 . Below, the results of a grazing incidence diffraction experiment dealing with defect scattering originating from near surface regions and from thin films are presented and compared.

For the purpose of reference a plain wafer has been measured first. This gives a reliable estimation of the influence of thermal diffuse scattering (TDS). Case 1 is realized by neutron irradiated silicon. Homogeneously distributed defects (density about $2000 \mathrm{ppm}$, neutron irradiation dose $5.8 \times 10^{18}$ neutrons $/ \mathrm{cm}^{-2}$ ) are obtained in this way. This sample belongs to the same category as the samples described in Ref. 13. The main part of this work deals with case 2: Here wafers were implanted with Co ions and subsequently annealed in different ways. In this

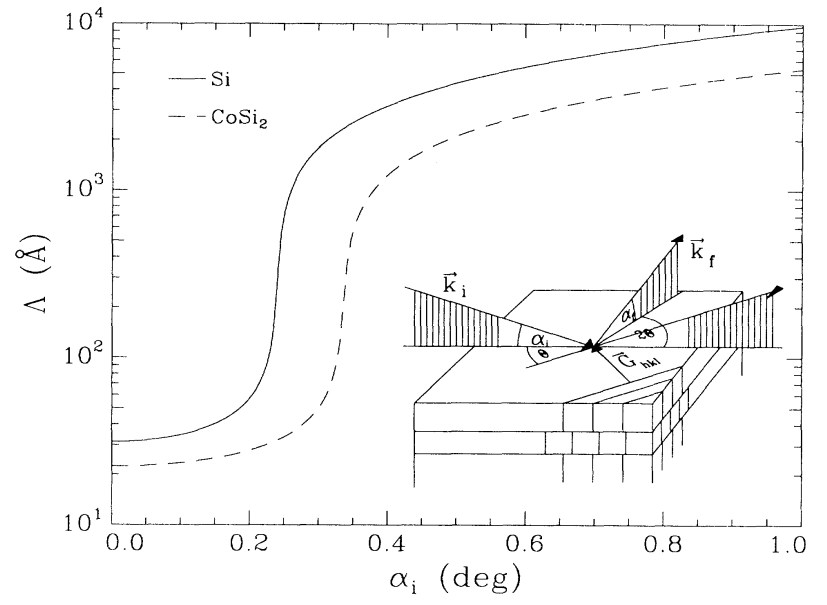

FIG. 1. Grazing incidence scattering geometry and scattering depth of $\mathrm{Si}$ (solid line) and $\mathrm{CoSi}_{2}$ (dashed line) with $\alpha_{i}=\alpha_{f}$

ion beam synthesis (IBS) a thin single-crystalline layer of metallic $\mathrm{CoSi}_{2}$ with rather smooth interfaces (roughness 10-30 $\AA$ ) which is buried underneath a single-crystalline layer of silicon is produced. ${ }^{18-20} \mathrm{CoSi}_{2}$ has fluorite structure with a lattice constant of $a_{0}=5.366 \AA$ which is only $1.2 \%$ below that of silicon. The silicide lattice is coherently coupled to the silicon substrate but even after annealing there remains an average lateral lattice mismatch of about $0.5 \%$.

The x-ray measurements were performed at the National Synchrotron Light Source (NSLS) at Brookhaven National Laboratory (BNL). The data were recorded on the $\mathrm{x}$-ray scattering beam line $\mathrm{X} 22 \mathrm{~B}$ with a wavelength $\lambda=1.65 \AA$. Figure 1 shows the grazing incidence geometry together with the angle dependent scattering depths. ${ }^{21,22}$ As the diagram shows the penetration depth of the $x$ rays can easily be limited to the surface silicon layer if the incidence angle is below $\alpha_{i} \approx 0.26^{\circ}$. By variation of $\alpha_{i}$ the scattering volume can be controlled which allows a depth profiling of the near surface part of the sample. In the same manner the buried silicide layer can be investigated by varying the incidence angle in the range $0.26^{\circ}<\alpha_{i}$.

\section{EXPERIMENTAL RESULTS}

The experimental results are presented under two aspects: (1) It will be shown that the calculations of Barabash and Krivoglaz are verified by our experiments. (2) By variation of the incidence angle the depth profile of the defect distribution will be analyzed.

Figure 2 shows rocking-scans $(\mathbf{q} \perp \mathbf{G})$ in the neighborhood of the in-plane 220 reflection of silicon for a variety of samples. An angle of incidence $\alpha_{i}=0.26^{\circ}$ was chosen and an $\alpha_{f}$ integration performed. As can be seen in Fig. 1, in this case the information depth is from the surface to about $1000 \AA$ below. As the layer thicknesses listed in Table I show, there is little scatter- 


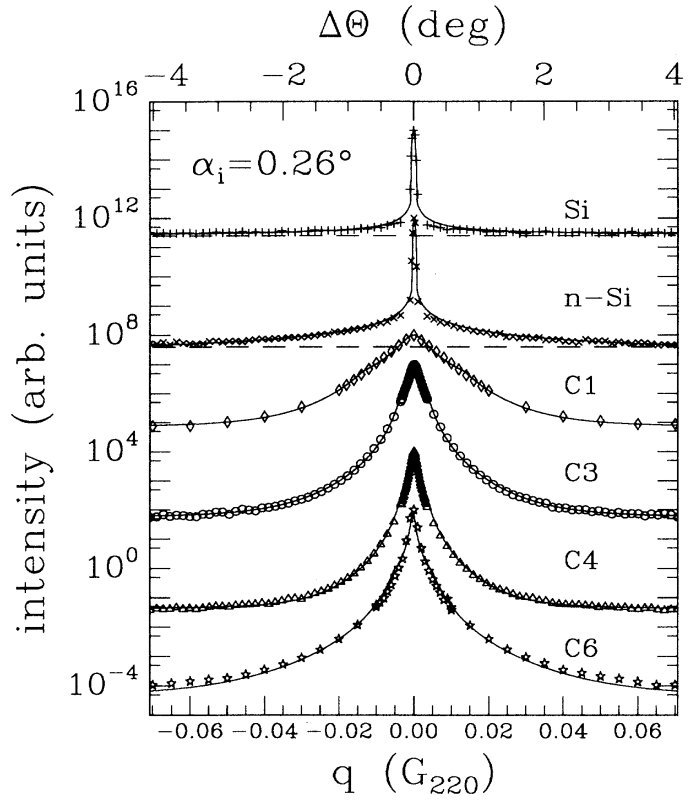



FIG. 2. The scattering around the in-plane 220 reflection of silicon $([1 \overline{1} 0]$ direction) for the different samples (see Table I). On the left the fit of the data to the theory is shown for the peak itself and the diffuse tails of the scattering, the dashed lines marking the background level. On the right the tail intensity is shown on a double logarithmic scale. This is to emphasize the different exponents of the power laws. ing from the underlying silicide layer. The data depicted in Table I have been gained by $\mathrm{x}$-ray reflectivity investigations at grazing angles. ${ }^{19}$ Figure 2 demonstrates that the shape of the rocking scans varies strongly with the sample. The near surface scattering from samples with a defect-containing volume (sample $n$-Si) contrasts that from the samples where the defects are restricted to a thin silicon film. The latter have a lattice constant different from the silicide layer (samples $C 1, C 3, C 4$, and C6). For comparison, an unirradiated reference sample is shown, too. The peaks from all thin film data are significantly broadened to values above the instrumental resolution whereas they are resolution limited for the samples $\mathrm{Si}$ and $n$-Si. The peak width depends on the defect density which is decreasing from sample $C 1$ to $C 6$. As the lines show, the data can be well described by a power law in the tails and a Gaussian ( $\mathrm{Si}, n$-Si), respectively, a Lorentzian $(C 1-C 6)$ in the central part. Double logarithmic plots of the intensity in the tails (Fig. 2) yield a $q^{-1}$ law for the neutron irradiated sample $(n-\mathrm{Si})$, whereas $q^{-4}$ is found for the IBS samples $(C 1-C 6)$.

The agreement between the theory of Barabash and Krivoglaz and the experiment is quite remarkable. Espe- cially the large difference between cases 1 and 2 can be confirmed persuasively.

The difference between in-plane reflections and conventional bulk reflections can be seen in Fig. 3. It displays the symmetric part of the diffuse scattering along the $q_{z}$ direction around the 004 reflection of $\mathrm{Si}$ in a $q$ range which shows no impact of the crystal truncation rod (see Ref. 23) and of the silicide 004 reflection. The double logarithmic presentation yields a straight line of slope -2 and hence demonstrates that the scattering follows the ordinary $q^{-2}$ law of Huang scattering [Eq. (1)]. As already mentioned this dependence is also contained in Eq. (3). These data also do show that the vast majority of the defects must be gathered in clusters whose radius is smaller than $25 \AA$. Otherwise the slope in Fig. 3 would have changed to -4 of the Stokes-Wilson scattering at $q>q_{0}=1 / R_{0}$ where $R_{0}$ is the radius of the clusters (e.g., $[9])$.

As can be seen from the scattering of the reference sample ( $\mathrm{Si}$ ) the influence of TDS can be neglected for our samples. This is not unexpected because the concentration of defects should be high and the data were taken at $T \approx 293 \mathrm{~K}$. This is significantly below the De-

TABLE I. Summarized information about the $\mathrm{CoSi}_{2}$ samples. The film thicknesses are cited from Ref. 19. The defect densities are normalized to the silicide layer of sample $C 1$.

\begin{tabular}{ccccc}
\hline \hline Sample & $C 1$ & $C 3$ & $C 4$ & $C 6$ \\
\hline Energy $(\mathrm{keV})$ & 200 & 200 & 200 & 200 \\
Dose $\left(\mathrm{cm}^{-2}\right)$ & $2 \times 10^{17}$ & $2 \times 10^{17}$ & $2 \times 10^{17}$ & $2 \times 10^{17}$ \\
Annealing & As implanted & RTA (2) & Furnace (2) & Furnace $(2)$ \\
Surface & $(001)$ & $(001)$ & $(001)$ & $(111)$ \\
$\mathrm{d}(\mathrm{Si})(\AA)$ & $820 \pm 23$ & $1067 \pm 0.6$ & $1070 \pm 2$ & $1030 \pm 1$ \\
$\mathrm{~d}\left(\mathrm{CoSi}_{2}\right)(\AA)$ & - & $750 \pm 1$ & $640 \pm 10$ & $692 \pm 0.3$ \\
Defects in Si & $34 \%$ & $4.0 \%$ & $2.3 \%$ & $1.7 \%$ \\
Defects in $\mathrm{CoSi}_{2}$ & $100 \%$ & $4.2 \%$ & $4.2 \%$ & $3.1 \%$ \\
\hline \hline
\end{tabular}




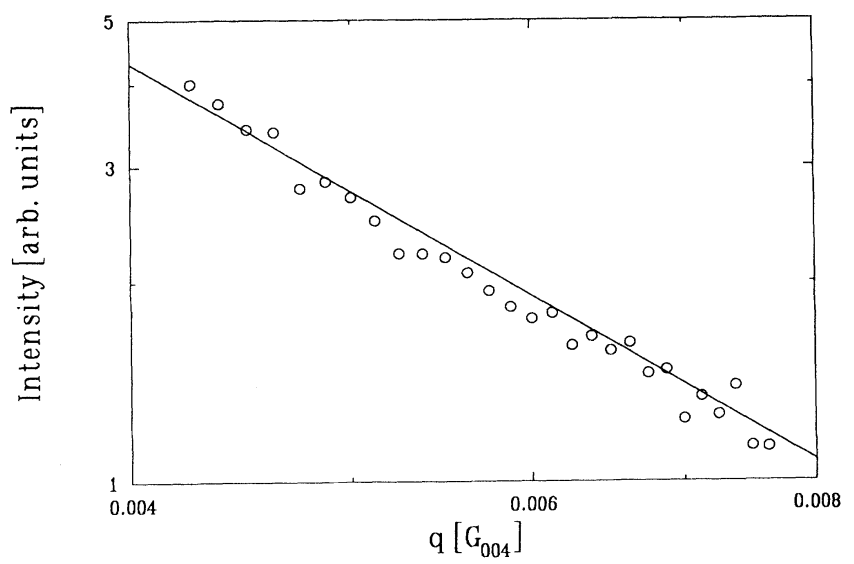

FIG. 3. Symmetric part of the diffuse scattering in the neighborhood of the $\mathrm{Si} 004$ bulk reflection of sample $C 3$ $\left(\mathbf{q}\left\|\mathbf{G}_{\mathbf{0 0 4}}\right\| \mathbf{q}_{\mathbf{z}}\right)$. The straight line has the $q^{-2}$ behavior of the Huang scattering.

bye temperatures of $\mathrm{Si}\left(\Theta_{D}=645 \mathrm{~K}\right)$ and $\mathrm{Co}\left(\Theta_{D}=\right.$ $445 \mathrm{~K})$. The thermal Debye-Waller factors of Co and $\mathrm{Si}$ at $T=300 \mathrm{~K}$ are nearly the same $\left[e^{-2 M_{D}}(\mathrm{Si})=0.914\right.$, $\left.e^{-2 M_{D}}(\mathrm{Si})=0.918\right]$. So the TDS of $\mathrm{CoSi}_{2}$ and $\mathrm{Si}$ should not differ by much. In conclusion the TDS of $\mathrm{Si}$ and $\mathrm{CoSi}_{2}$ which is proportional to $\left[1-\exp \left(-M_{D}\right)\right]$ should be negligible.

We now turn to the depth profiling of the defect distribution which can be controlled by the incidence angle $\alpha_{i}$ (Fig. 1). Figure 4 shows several $\alpha_{f}$-integrated Bragg

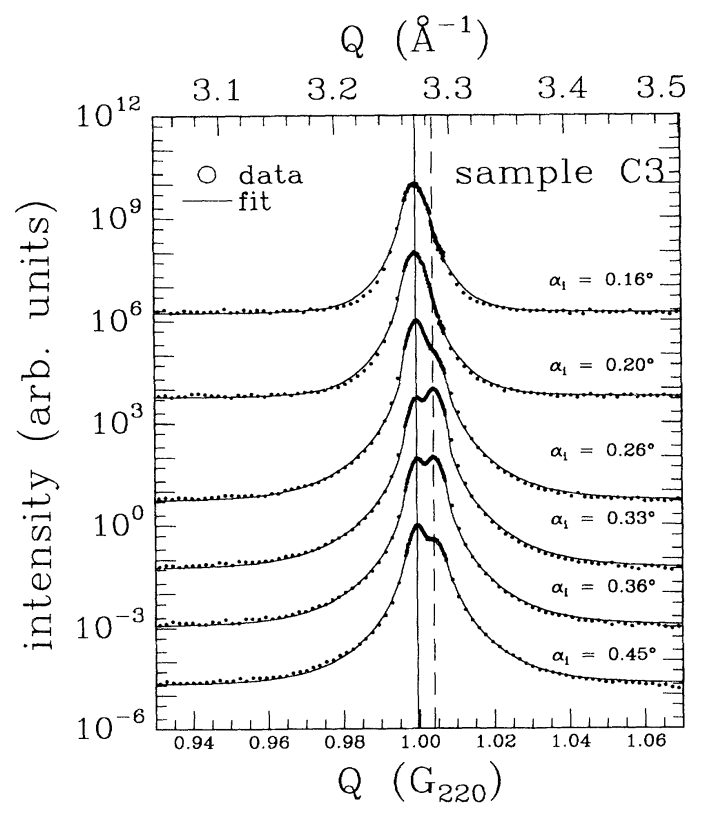

FIG. 4. The scattering in [110] direction for one of the IBS samples together with a fit based on case 2 of the theory of Barabash and Krivoglaz. For $\alpha_{i}>0.26^{\circ}$ the scattering depth is large enough to illuminate the silicide layer. The full line marks the position of the $\mathrm{Si} 220$ reflection, the dashed one that of the $\mathrm{CoSi}_{2} 220$ reflection. scans $(\mathbf{q} \| \mathbf{G})$ at different angles $\alpha_{i}$. Below $\alpha_{i}=0.26^{\circ}$, only the silicon 220 reflection is visible. At larger $\alpha_{i}$ and, correspondingly, at greater penetration depths $\Lambda$, the silicide reflection grows in intensity and reaches its highest value when the whole silicide film is illuminated (compare Fig. 1). As the lines of the fits show, the theory of Barabash and Krivoglaz (case 2) describes the data very well.

With x-ray scattering as a probe, inevitably all information is obtained by integrating over macroscopic parts of the sample. Here, the scattering is from a volume composed of a surface area of about $1 \mathrm{~mm}^{2}$ and a depth ranging from 20 to $10000 \AA$ (Fig. 1). In the case of the neutron irradiated sample the amount of defects contributing


FIG. 5. (a) Depth scaling of the diffuse scattering. The diffuse scattering is normalized to the corresponding Bragg intensity. (b) Depth scaling of the half widths of the reflections. The width of the neutron irradiated sample is resolution limited. In both cases the $\Lambda$ scale of the scattering depth is calculated from the angles $\alpha_{i}$ and $\alpha_{f}$. (Cross, neutron irradiated $\mathrm{Si}$; circle, $\mathrm{Si}$ of sample $C 3$; full circle, $\mathrm{CoSi}_{2}$ of sample $C 3$; triangle, $\mathrm{Si}$ of sample $C 4$; full triangle, $\mathrm{CoSi}_{2}$ of sample C4.) The lines are guides to the eye only. 
to the diffuse scattering is increasing proportionally to the thickness of the illuminated surface layer. Therefore, the diffuse scattering should increase proportionally to the scattering depth $\Lambda$. This is verified by Fig. 5, where the angles $\alpha_{i}$ and $\alpha_{f}$ have been converted to a scattering depth $\Lambda$.

In the case of the four silicide samples $(C 1-C 6)$ the intensity is also increasing with the number of lattice layers contributing to the scattering (both $\mathrm{Si}$ and $\mathrm{CoSi}_{2}$ layer). Different from the experiment with the bulk crystal there is a huge amount of additional diffuse scattering from the silicon surface and the interface $\mathrm{Si} / \mathrm{CoSi}_{2}$ : The diffuse intensity peaks in the range $\Lambda<100 \AA$ and 1000 $<\Lambda<1100 \AA$. The same information is provided by the widths of the peaks. The width of the silicide reflections is much greater if only the interface area is illuminated (Fig. 5) whereas the widths of the silicon reflections do not vary as markedly.

The information from the peak widths and the diffuse scattering can be used to compare the defect concentration in the various silicon and silicide layers. Table I shows the results normalized to the diffuse scattering of the silicide layer of sample $C 1$, which has the highest defect density, taken as $100 \%$. The half widths of the reflections give within error the same results. The sample with [111] surface orientation $(C 6)$ has the lowest defect concentrations. The annealing reduces the defect densities to about the same values in the silicon layer and the silicide. The standard furnace heating which lasts about 100 times as long as the rapid thermal annealing process seems to reduce the number of defects somewhat more efficiently.

\section{CONCLUSIONS}

In summary this investigation shows that the scattering of $\mathrm{x}$ rays by thin films with defects has to be evaluated in a quite different manner compared to scattering from bulk crystals (e.g., Ref. 9). Moreover, it becomes important in which way these films are grown onto a substrate. The experimental findings confirm the results of the calculations of Barabash and Krivoglaz.

In addition, the use of grazing incidence diffraction provides the possibility of probing the defect contributions from different depths of a sample. Especially the defect densities at the interfaces and in sandwiched films can be separated.

\section{ACKNOWLEDGMENTS}

The project was supported by the BMFT under Contract Nos. 05401ABI2 and 5FKABB/B.4-D01. Many helpful discussions with J. Stettner, M. Tolan, and B. Burandt are gratefully acknowledged as well as the enormous hospitality at Brookhaven.

\footnotetext{
*Present address: HASYLAB at DESY, D-22603 Hamburg, Germany.

${ }^{1}$ R. I. Barabash and M. A. Krivoglaz, Sov. Phys. Solid State 29, 1768 (1987).

${ }^{2}$ W. Fenzl and J. Peisl, Phys. Rev. Lett. 54, 2064 (1985).

${ }^{3}$ H. Dosch, L. Mailänder, A. Lied, J. Peisl, F. Grey, R. L. Johnson, and S. Krummacher, Phys. Rev. Lett. 60, 2382 (1988).

${ }^{4}$ B. Burandt, W. Press, and S. Haussühl, Phys. Rev. Lett. 71, 1188 (1993).

${ }^{5}$ K. Huang and H. H. Wills, Proc. R. Soc. London, Ser. A 190, 102 (1947).

${ }^{6}$ M. A. Krivoglaz, Theory of $X-R a y$ and Thermal-Neutron Scattering by Real Crystals (Plenum, New York, 1969).

${ }^{7}$ P. H. Dederichs, Met. Phys. F3, 471 (1973).

${ }^{8}$ H. Trinkaus, Phys. Status Solidi B 51, 307 (1972).

${ }^{9}$ P. Ehrhart and W. Schilling, Phys. Rev. B 8, 2604 (1973).

${ }^{10}$ P. Ehrhart, J. Nucl. Mater. 69 \& 70, 200 (1978).

${ }^{11}$ S. Dietrich and W. Fenzl, Phys. Rev. B 39, 8873 (1989).
}

${ }^{12}$ S. Dietrich and W. Fenzl, Phys. Rev. B 39, 8900 (1989).

${ }^{13}$ S. Grotehans, G. Wallner, E. Burkel, H. Metzger, J. Peisl, and H. Wagner, Phys. Rev. B 39, 8450 (1989).

${ }^{14}$ A. Yu. Belov and V. M. Kaganer, Phys. Met. 9, 665 (1990).

${ }^{15}$ R. Bloch, D. Bahr, J. Olde, L. Brügemann, and W. Press, Phys. Rev. B 42, 5093 (1990).

${ }^{16}$ V. Holý, J. Kubena, and Z. Bochiníĉek, J. Appl. Phys. 70, 3537 (1991).

${ }^{17}$ V. Holý, Appl. Phys. A 58, 173 (1994).

${ }^{18}$ S. Mantl, Mater. Sci. Rep. 8, 1 (1992).

${ }^{19}$ D. Bahr, W. Press, R. Jebasinski, and S. Mantl, Phys. Rev. B 47, 4385 (1993).

${ }^{20}$ M. Müller, D. Bahr, W. Press, R. Jebasinski, and S. Mantl, J. Appl. Phys. 74, 1590 (1993).

${ }^{21}$ H. Dosch, Phys. Rev. B 35, 2137 (1987).

${ }^{22} \mathrm{H}$. Dosch, Critical Phenomena at Surfaces and Interfaces (Evanescent X-Ray and Neutron Scattering), Springer Tracts in Modern Physics Vol. 126 (Springer, Berlin, 1992).

${ }^{23}$ I. K. Robinson, Phys. Rev. B 33, 3830 (1986). 